\title{
Something in Nothing: Negative Space in the Clinician-Patient Relationship
}

Stephen A. Buetow, PhD

Department of General Practice and Primary Health Care, University of Auckland, Auckland, New Zealand
Conflicts of interest: none reported

\section{CORRESPONDING AUTHOR}

Stephen A Buetow, PhD Department of General Practice and Primary Health Care University of Auckland Private Bag 92019

Auckland Mail Centre Auckland 1142, New Zealand

s.buetow@auckland.ac.nz

\begin{abstract}
Clinicians can easily miss the importance of how what is not present gives depth, perspective, and clues to the real meaning of social action in clinical encounters. This essay addresses examples of commonly overlooked forms of this concept of negative space in the clinician-patient relationship. The examples are negative physical space, which denotes the physical distances that separate clinicians and patients during face-to-face encounters; negative communicative space, which refers to how nonverbal and verbal communication can signify information not exchanged during these visits; and negative longitudinal space, which describes pauses over time and includes what is not said between clinicians and patients between visits. Discussed is how the awareness of these different spaces helps us to let go of our preconceptions, to experience what is there rather than what we expect to find, and to use space sensitively to improve interactions with patients.
\end{abstract}

Ann Fam Med 2009;7:80-83. DOI: 10.1370/afm.914.

$\Lambda$ $\mathrm{rt}$ provides lessons that are relevant to family practice and include the need to look closely without preconceptions. ${ }^{1}$ Close observation is important because there is a common tendency to focus on positive space, which is the space occupied by subjects. It is easy to miss the negative space, which in art is the space between or around subjects. Art teaches us that if we ignore the negative space, we risk seeing only the positive space we expect to see, rather than what is there. Awareness of negative space enables us to let go of our presumptions and be open to new possibilities. For example, a well-known illustration of negative space is shown in Figure 1; which first reveals a vase but, on reflection, also depicts 2 faces in profile.

The concept of negative space signifies in medicine what is not seen, not heard, not felt, or otherwise not done or experienced. Negative space frames and provides context for what is present, for example, during clinician-patient interactions. The context of these interactions is incomplete without alertness to negative space, because in the practice of medicine clinicians can easily fail to notice-and be responsive to-what is absent in perceiving what is at hand.

As health professionals, we need to be aware of what is not, in the midst of what is, so we can move beyond what we expect, or take for granted, in interpreting the positive spaces that clinicians and patients occupy. This essay focuses on the concept of negative space in clinicianpatient interactions. Specifically, it considers 3 neglected forms of negative space in these encounters: (1) negative physical space, which denotes the physical distances that separate the clinician and patient during their face-to-face encounters; (2) negative communicative space, which refers to how nonverbal and verbal communication can each signify information not exchanged during these visits; and (3) negative longitudinal space, which describes pauses over time, including what the clinician and patient 


\section{Figure 1. Rubin's vase.}

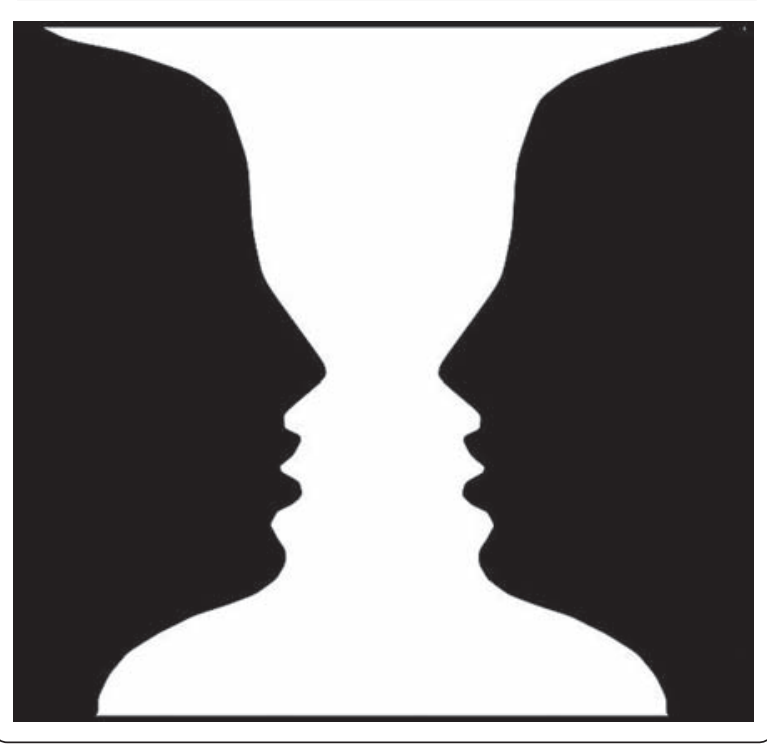

do not say to each other between visits. I discuss how our alertness to, understanding of, and sensitive use of these different spaces can improve the delivery of clinical care in family medicine.

\section{Negative Physical Space}

Communication during visits is enhanced when the clinician is aware of and appropriately uses empty physical spaces to meet patients', as well as and their own, expectations. These spaces take 3 forms: informal space, fixed space, and semifixed feature space. Requirements of the clinician and patient for informal space define 4 territorial zones of social interaction ${ }^{2}$ : intimate distance, which includes the use of touch and characterizes physical examinations ${ }^{3,4}$; personal distance, which is less close but allows a soft voice and private discussion, social distance, which suits nonpersonal exchanges in full voice; and public distance, which a clinician might use to call on patients in the waiting room.

These zones should fit the activities undertaken within them. Most patients favor clinicians who appropriately minimize the use of personal distance to indicate attention and interest, for example by adopting body positions or movements such as open arms, head nodding, and leaning forward. ${ }^{5-7}$ Intimate distances are especially suited to producing a sense of interpersonal connectedness, but they can be intrusive, unsettling and increase the risk of contact that violates professional boundaries. Meanwhile, overlarge negative physical spaces risk unwanted, interpersonal detachment. ${ }^{8}$

Within and around the zones of informal interaction, configurations of objects and people also define negative physical spaces that can influence face-to-face interactions. These spaces may be fixed and static, as defined by such immotile features as walls and some types of furniture, or they may be semifixed and dynamic, as, for example, movable furniture. The latter spaces enhance opportunities to influence social action. For example, within the examination room zones of personal distance and social distance, areas of emptiness may be consciously and productively created around furniture to facilitate access and movement and to increase available light and room for sitting. Empty areas, as on walls and stands, and an absence of color can also be used to emphasize positive spaces, such as those occupied by leaflets and posters. Another example is using chairs of the same height ${ }_{i}$ reducing the physical space between the clinician and the patient minimizes power differences and facilitates interpersonal communication. Consider also the spaces created by seating arrangements. For example, sitting corner to corner is less formal and more flexible and welcoming than sitting at a desk across from the patient.

Cultural and social attributes, norms, and rituals can influence the size, meaning, and use of informal and feature spaces. ${ }^{2}$ For example, those in contact cultures, such as Latin Americans and southern Europeans, tend to be more comfortable with informal interactions in physically closer spaces than those in noncontact cultures, such as Asians and northern Europeans. ${ }^{9}$ Where possible, it is best to ascertain what is acceptable to each patient than to make assumptions based on group attributes. Feature spaces, however, can be fixed or semifixed features to meet the needs of the clinician and most patients $s_{i}$ for example, the application of feng shui principles is considered appropriate by many Chinese people but not by some modern Christians. ${ }^{10}$

\section{Negative Communicative Space}

Negative communicative space, which refers to information that is not exchanged, can take 3 forms in the clinical encounter. The first is silence, as abstention from speech. The second form comprises other nonverbal actions that exclude some information. For example, eye aversion by patients can both reveal and exclude information. It shuts out some of the information we can get from a patient's face, including eyes. In general, we are conscious of the look of the other person rather than of his or her eyes." Nevertheless, if we cannot see a patient's eyes, we miss the information that eyes can reveal, for example, surprise (eyes widen), happiness (eyes glow), or interest and cognitive workload (eyes dilate). The third is verbal communication, which can sometimes signify negative communicative space. For example, all too frequently physicians restrict the communicative space available 
to their patients by verbally interrupting them, ${ }^{12,13}$ despite research ${ }^{14,15}$ showing that allowing patients to complete their opening statement requires little time and does not disrupt the visit. Clinicians and patients can also produce conversational silences by excluding the discussion of difficult topics, such as illicit drug use, sexuality, family violence, ${ }^{16}$ and not telling the complete truth. ${ }^{17}$

Although such behaviors can hinder face-to-face communication and social connectedness ${ }^{18}$ it is important to avoid preconceptions when interpreting their meanings and importance. For example, silence can support social interaction by indicating attentiveness and giving direction to taking turns. The meanings of silence vary culturally, in that quiescence may or may not suggest interest, engagement, and assent. ${ }^{19}$ Silence can strategically facilitate reflection, problem solving, and an expression and attunement of feelings. ${ }^{20,21}$ It can also put social pressure on patients to speak.

Silence and related verbal and nonverbal actions, such as emotional detachment, may be intended to minimize the risk of injury to the clinician, the patient, or both. These behaviors, however, can produce a reactive sense of loneness or abandonment in patients, reducing the quality of decision making and the health care experience. ${ }^{22}$

It is important, therefore, to understand how negative communicative space helps to construct, and is constructed by, the discourse around it. To this end, clinicians need to reflect on their own communication; to question individual patients sensitively as to the meanings of what they, as patients, communicate verbally and nonverbally during visits; to gauge mutual tolerance for these behaviors; and to use silence and associated verbal and nonverbal behaviors in ways that do not preclude a discussion of delicate issues or an acknowledgment of uncertainty and difference.

\section{Negative Longitudinal Spaces}

Negative longitudinal spaces include intervisit spaces, which are the periods of time that physically separate clinical encounters. Negative spaces between visits by the same patient signify temporal discontinuities in the delivery of formal care. The greater the spaces, the greater the potential need to update patient details. Large spaces, resulting from infrequent visits, have also been associated with a reduction in the continuity that clinicians and informal caregivers may each provide, ${ }^{23}$ and they can threaten achievement of the therapeutic goals set for many chronic illnesses. ${ }^{24}$

The meanings of spaces between visits, however, also depend on expectations. Clinics interpret the interval since a patient's last visit in the context of that patient's usual attendance pattern. For example, if a patient were scheduled to return in 3 months but had not revisited by 5 months, the clinic might reasonably wish to know why. In addition, lengthened intervisit spaces have meaning for patients. For example, patients who cannot access family medical care as soon as they want or expect for a nonurgent problem might be encouraged to seek this care elsewhere, such as from an emergency department.

Nevertheless, some time apart is important for clinicians and patients. It helps clinics manage patient demand, and in family medicine, where many health conditions are self-limiting, it helps patients learn selfcare and how to manage chronic conditions. Clinically spaces between visits can also be necessary, as with wait-and-see strategies. Negative longitudinal spaces, however, can be costly if they do not improve health outcomes relative to infrequent visits; they can be unsafe if they reduce clinical detachment, mask gradual clinical changes (such as the insidious onset of classical hypothyroidism), and reduce access for other patients; and they can indicate a health problem, including a somatoform disorder or atypical perception of when medical care is necessary.

To minimize the discontinuities between visits, patients and clinicians use the telephone, mail, and, increasingly, electronic communication, such as email ${ }^{25,26}$ and text messaging on mobile telephones. ${ }^{27,28}$ E-communication can help patients and clinicians stay in contact, improve continuity, and transfer information to facilitate case management, especially for chronic conditions. ${ }^{29}$ Asynchronous e-communication facilitates reflection and delegation. Clinicians can now access guidelines to help negotiate rules around patient e-communications ${ }^{30}$; and a number of health plans are reimbursing clinicians for certain types of e-communications with insured patients.

In conclusion, medical care tends to focus on the positive spaces occupied by participants. Clinicians' expectations of these spaces influence what they perceive, and they need to be aware of and manage their tendency for selective perception. To these ends, I have suggested an unmet need for clinicians to look carefully and without preconceptions at the whole picture by attending to the negative spaces in the clinician-patient relationship. This need will be met only if clinicians know how and expect to look for it. Accordingly, I have explored different forms of negative space and considered how they can be managed, as windows of opportunity, to put into context and improve clinical encounters.

These ideas, however, warrant further theoretical development. Also needed is empirical research: first, to assess the use and influence of negative spaces in creating meaning in clinician-patient interactions; 
and second, to evaluate the safety and effectiveness of interventions (for example, in education) to help manage the spaces. Initial priorities could include investigations into how clinicians and patients can best negotiate the physical distances that frame their interactions, clarify and manage the meanings of negative communicative spaces in clinical encounters, become attuned to the meanings that they each attach to intervisit spaces, cooperate to transfer information between visits, and identify appropriate return visit intervals. Dissemination strategies should be designed from the outset to raise awareness of research plans and developments and to facilitate the translation of positive research findings into clinical practice.

To read or post commentaries in response to this article, see it online at http://www.annfammed.org/cgi/content/full/7/1//80.

Submitted November 22, 2007; submitted, revised, May 13, 2008; accepted June 2, 2008.

Key words: Negative space; physician-patient relations; patient care

Acknowledgments: I am grateful to 2 external peer reviewers, the editors, and Tim Kenealy for their helpful comments on earlier versions of this essay.

\section{References}

1. Sweeney B. Postcard 7... Is art the finest teacher? Fine art and medicine. Br J Gen Pract. 2004;54(498):70-71.

2. Hall E. The Hidden Dimension. Garden City: Doubleday; 1966.

3. McWhinney I. A Textbook of Family Medicine. 2nd ed. New York, NY: Oxford University Press; 1997.

4. Makoul G, Zick A, Green M. An evidence-based perspective on greetings in medical encounters. Arch Intern Med. 2007;167(11):1172-1176.

5. Larsen KM, Smith CK. Assessment of nonverbal communication in the patient-physician interview. J Fam Pract. 1981;12(3):481-488.

6. Harrigan J, Rosenthal R. Physicians head and body positions as determinants of perceived rapport. J Appl Soc Psychol. 1983;13(6): 496-509.

7. Pandhi N, Bowers B, Chen F-P. A comfortable relationship: a patientderived dimension of ongoing care. Fam Med. 2007;39(4):266-273.

8. Enbon JA, Thomas CD. Evaluation of sexual misconduct complaints: the Oregon Board of Medical Examiners, 1991 to 1995. Am J Obstet Gynecol. 1997;176(6):1340-1348.

9. Remland MS, Jones TS, Brinkman H. Interpersonal distance, body orientation and touch: effects of culture, gender, and age. J Soc Psychol. 1995;135(3):281-297.
10. Montenegrom M. Feng shui. New dimensions in design. Christian Res J. 2003;26(1). http://cana.userworld.com/cana_fengShui4.html.

11. Sartre J-P. Being and Nothingness. London: Routledge, 1958.

12. Beckman HB, Frankel RM. The effect of physician behavior on the collection of data. Ann Intern Med. 1984;101(5):692-696.

13. Marvel MK, Epstein RM, Flowers K, Beckman HB. Soliciting the patient's agenda: have we improved? JAMA. 1999;281(3):283-287.

14. Langewitz W, Denz M, Keller A, Kiss A, Ruttimann S, Wossmer B. Spontaneous talking time at start of consultation in outpatient clinic: cohort study. BMJ. 2002;325(7366):682-683.

15. Rabinowitz I, Luzzati R, Tamir A, Reis S. Length of patient's monologue, rate of completion, and relation to other components of the clinical encounter: observational intervention study in primary care. BMJ. 2004;328(7438):501-502.

16. Bonomi AE, Allen DG, Holt VL. Conversational silence, coercion equality: the role of language in influencing who gets identified as abused. Soc Sci Med. 2006;62(9):2258-2266.

17. Clafferty RA, McCabe E, Brown KW. Conspiracy of silence? Telling patients with schizophrenia their diagnosis. Psychiatr Bull. 2001;25: 336-339.

18. Ness DE, Kiesling SF. Language and connectedness in the medical and psychiatric interview. Patient Educ Couns. 2007;68(2):139-144.

19. Lane RC, Koetting MG, Bishop J. Silence as communication in psychodynamic psychotherapy. Clin Psychol Rev. 2002;22(7):1091-1104.

20. Elson M. Silence, its use and abuse: a view from self-psychology. Clin Soc Work J. 2001;29(4):351-360.

21. Hill CE, Thompson BJ, Ladany N. Therapist use of silence in therapy: a survey. J lin Psychol. 2003;59(4):513-524.

22. Bugge C, Entwistle V, Watt I. The significance for decision-making of information that is not exchanged by patients and health professionals during consultations. Soc Sci Med. 2006;63(8):2065-2078.

23. Pilotto LS, McCallum J, Raymond C, McGilchrist C, Veale BM. Sequential continuity of care by general practitioners: which patients change doctor? Med J Aust. 1996;164(8):463-466.

24. Schectman G, Barnas G, Laud P, Cantwell L, Horton M, Zarling EJ. Prolonging the return visit interval in primary care. Am J Med. 2005;118(4):393-399.

25. Pal B. E-mail contact between doctor and patient. BMJ. 1999; 318(7195):1428.

26. Car J, Sheikh A. E-mail consultations in health care: 1-scope and effectiveness. BMJ. 2004;329(7463):435-438.

27. Rodgers A, Corbett T, Bramley D, et al. Do u smoke after txt? Results of a randomised trial of smoking cessation using mobile phone text messaging. Tob Control. 2005;14(4):255-261.

28. Dhar J, Leggat C, Bonas S. Texting - a revolution in sexual health communication. Int J STD AIDS. 2006;17(6):375-377.

29. Holman $\mathrm{H}$, Lorig K. Patients as partners in managing chronic disease. BMJ. 2000;320(7234):526-527.

30. Kane BA, Sands DL. Guidelines for the clinical use of electronic mail with patients. JAMA. 1998;280(15):1303-1304. 